\title{
RIQUEZA E COMPOSIÇÃO FLORÍSTICA DA COMUNIDADE DE SAMAMBAIAS NA MATA CILIAR DO RIO CADEIA, RIO GRANDE DO SUL, BRASIL
}

\author{
RICHNESS AND FLORISTIC COMPOSITION OF THE FERN COMMUNITY IN RIPARIAN \\ FOREST OF THE RIVER 'CADEIA', IN RIO GRANDE DO SUL STATE, BRAZIL
}

\author{
Ivanete Teresinha Mallmann ${ }^{1}$ Jairo Lizandro Schmitt ${ }^{2}$
}

\begin{abstract}
RESUMO
Este trabalho analisou a riqueza e a composição da comunidade de samambaias em fragmentos de mata ciliar do rio Cadeia, sob diferentes níveis de antropização, em Santa Maria do Herval, Rio Grande do Sul, Brasil. Foram alocadas 120 parcelas distribuídas equitativamente em três fragmentos (FI, FII e FIII), sendo inventariadas todas as espécies e registrada a riqueza por unidade amostral. A composição florística entre os fragmentos foi comparada empregando-se o índice de Jaccard e a distribuição espacial das parcelas foi avaliada por meio de escalonamento multidimensional. Os dados de riqueza foram apresentados utilizando-se curvas de rarefação baseadas em amostras e estimadores não paramétricos de diversidade. Foram encontradas 40 espécies, distribuídas em 13 famílias. A maior similaridade florística ocorreu entre o FI e o FII. As parcelas do FI formaram o agrupamento mais definido e apresentaram o maior número de espécies exclusivas. A curva de rarefação para a amostra total se aproximou da assíntota e os estimadores apontaram no máximo 45 espécies, evidenciando que a maioria das espécies foi inventariada na mata ciliar. Um gradiente decrescente de riqueza média por parcela foi observado à medida que aumenta a urbanização no habitat matriz dos fragmentos. Esses resultados constituem uma base de dados a ser utilizada em manejo, conservação e reflorestamento de matas ciliares degradadas. Eles podem ser comparados diretamente aos resultados obtidos em outros trabalhos que utilizam rarefação e estimativas de riqueza, o que não é possível com muitos dos inventários realizados no Brasil.
\end{abstract}

Palavras-chave: inventário florístico; rarefação; estimadores de riqueza; conservação.

\section{ABSTRACT}

The present study analyzed richness and specific composition of the fern community in fragments from the riparian forest of river 'Cadeia', under different levels of human impact, in Santa Maria do Herval, Rio Grande do Sul state, Brazil. An amount of 120 sample units were delimited, equitably distributed in three fragments (FI, II and III) in which all species were surveyed and the richness was recorded. The floristic composition among fragments was compared using Jaccard's index and spatial distribution of units was evaluated through multidimensional scaling. Richness data were presented in the form of rarefaction curves based on samples and non-parametric diversity estimators. A total of 40 species were found, belonging to 13 families. The greater floristic similarity was between FI and FII. Sample units from FI formed the most defined grouping and they had more exclusive species than the others. The rarefaction curve for the total sampling almost reached the asymptote and estimators indicated a maximum of 45 species, which means that the majority of species was surveyed at the study site. A decreasing gradient of mean richness per unit was observed as the urbanization increased in the matrix habitat of the fragments. These results form a data base to be used in management, conservation and reforestation measures in degraded riparian forests. They can be directly compared to results from other studies that used rarefaction and richness estimators, which is not possible to do with many of the surveys accomplished in Brazil so far.

Keywords: floristic survey; rarefaction; richness estimators; conservation.

1 Bióloga, Msc., Programa de Pós-Graduação em Qualidade Ambiental, Federação de Estabelecimento de Ensino Superior Novo Hamburgo (Universidade Feevale), ERS 239, n. 2755, CEP 93352-000, Novo Hamburgo (RS), Brasil. ivamallmann@terra.com.br

2 Biólogo, Dr., Professor Titular do Programa de Pós-Graduação em Qualidade Ambiental, Federação de Estabelecimento de Ensino Superior Novo Hamburgo (Universidade Feevale), ERS 239, n. 2755, CEP 93352-000, Novo Hamburgo (RS), Brasil. jairols@,feevale.br

Recebido para publicação em 24/09/2010 e aceito em 2/04/2013 


\section{INTRODUÇÃO}

A massa de vegetação que se forma naturalmente às margens dos rios constitui as matas ciliares (MUELLER, 1996). Elas protegem o curso d'água, a biodiversidade e o solo, contribuem positivamente para a produção primária do sistema lótico e funcionam como corredores ecológicos (GREGORY et al., 1992; OLIVEIRA-FILHO e RATTER, 1995; MUELLER, 1996). No entanto, a degradação das matas ciliares continua sendo, principalmente, consequência da expansão agrícola desordenada (RODRIGUES e GANDOLFI, 2000). Além disso, a perda gradativa da qualidade ambiental expande-se ao longo das bacias hidrográficas, associada também ao adensamento populacional em suas áreas (BARELLA et al., 2000).

Estudos florísticos em matas ciliares têm apontado heterogeneidade na composição e estruturação das espécies, tanto em escala geográfica quanto local (RIBEIRO-FILHO et al., 2009), sendo que inclusive remanescentes próximos apresentam similaridade baixa (OLIVEIRA-FILHO e RATTER, 1995). A interação complexa e diferenciada na intensidade, no tempo e no espaço de fatores físicos (relevo, profundidade do lençol freático) e biológicos (grau de conservação, tamanho da faixa ciliar, habitat matriz) é determinante da heterogeneidade florística das matas ciliares (RODRIGUES e NAVE, 2001).

Estas formações florestais que acompanham os cursos d'água ou o entorno de nascentes podem apresentar elevada riqueza específica de samambaias e licófitas, em território brasileiro (WINDISCH, 1996). Essas plantas, no Brasil, constituem um grupo com 1.176 espécies (PRADO e SYLVESTRE, 2010), das quais 32 espécies de licófitas (LORSCHEITTER et al., 1998; 2009) e 322 de samambaias (FALAVIGNA, 2002) estão distribuídas no Rio Grande do Sul. As áreas florestadas constituem os ambientes de maior riqueza de samambaias (SEHNEM, 1979) e licófitas, no Estado. Foram publicados, no Brasil, levantamentos locais de riqueza e composição específica de samambaias e licófitas, realizados em diferentes tipos vegetacionais ribeirinhos, com diferentes estados de conservação, utilizando metodologias distintas. Rodrigues et al. (2004) encontraram oito espécies em floresta de igapó, na bacia do rio Guamá, Belém, no estado do Pará. No Mato Grosso, Athayde Filho e Windisch (2003) identificaram 19 e 10 espécies de samambaias e licófitas, respectivamente, em mata de galeria e vereda, na Reserva Biológica Mário Viana, Nova Xavantina; Athayde Filho e Agostinho (2005) registraram um total de 11 espécies, em duas veredas, no município de Campinápolis; Athayde Filho e Felizardo (2007) inventariaram 26 espécies, em três segmentos florestais do rio Pindaíba, nos municípios de Barra do Garças, Araguaiana e Nova Xavantina; e Kreutz e Athayde Filho (2009) registraram 14 espécies, em quatro ordens do córrego Caveira, Barra do Garças.

No Rio Grande do Sul, Diesel e Siqueira (1991) realizaram levantamento fitossociológico do estrato herbáceo arbustivo, em três áreas de mata ripária da bacia do rio dos Sinos e registraram um total de 71 espécies das quais 24 foram samambaias. Apesar de sua evidente contribuição na composição florística das formações ribeirinhas, ainda são escassos os estudos sobre as comunidades de samambaias e licófitas, em decorrência de que muitos pesquisadores enfocam apenas angiospermas arbóreas (CAMPOS e LANDGRAF, 2001).

Para otimizar a utilização dos resultados de inventários biológicos na tomada de decisões em conservação, é fundamental obter dados de riqueza comparáveis, mesmo entre conjunto de dados obtidos com metodologias e esforço amostral distintos (SANTOS, 2006). No entanto, no Brasil, os estudos de descrição de comunidades vegetais continuam a ser realizados sem uma padronização de dados, tornando a grande quantidade de informações gerada de difícil comparação (SONEGO et al., 2007). Uma das formas para se alcançar isso é a utilização de curvas de rarefação e estimadores de riqueza, a partir de dados amostrais (GOTELLI e COLWELL, 2001).

O objetivo do presente estudo foi analisar a riqueza e a composição da comunidade de samambaias na mata ciliar do rio Cadeia, em Santa Maria do Herval, Rio Grande do Sul, Brasil descrevendo a forma de vida e de crescimento, bem como o substrato preferencial das espécies. Os dados foram gerados de modo que poderão ser comparados com outros estudos futuros e constituem uma base de dados florísticos a ser utilizada em manejo, conservação e reflorestamento de matas ciliares degradadas.

\section{MATERIAL E MÉTODOS}

\section{Área de estudo}

O rio Cadeia é um dos seis maiores afluentes do rio Caí, principal curso d'água da bacia hi- 
drográfica do Caí, no estado do Rio Grande do Sul, Brasil. De acordo com a classificação de Koeppen, o clima na bacia é do tipo Cfa, ou seja, temperado úmido $(\mathrm{C})$ com ocorrência de chuvas durante todos os meses do ano (f), sendo a temperatura média do mês mais quente superior a $22^{\circ} \mathrm{C}$ (a) (MORENO, 1961). A vegetação dominante pertence à unidade fitoecológica da Floresta Estacional Semidecidual (TEIXEIRA et al., 1986).

No município de Santa Maria do Herval, $37,58 \%$ da área de preservação permanente do rio Cadeia está sem a cobertura vegetal prevista pelo Código Florestal (SARMENTO et al., 2001). Nesse município, foram selecionados três fragmentos de mata ciliar (Figura 1) para a realização do estudo:

Fragmento I (FI): situa-se no extremo leste de Santa Maria do Herval, distante $11 \mathrm{~km}$ do centro da cidade $\left(29^{\circ} 31^{\prime} 1.02\right.$ 'S e 5054'46.78'W, $509 \mathrm{~m}$ de altitude). A mata ciliar tem $160 \mathrm{~m}$ de largura sendo que dados históricos indicam que não há ação antrópica intensa sobre a vegetação há cerca de 70 anos. O FI está inserido em uma matriz rural, com baixa densidade demográfica.
Fragmento II (FII): situa-se a $2,4 \mathrm{~km}$ de distância do centro do município (29³0'14.20"S e $50^{\circ} 58^{\prime} 28.13^{\prime \prime} \mathrm{W}, 399 \mathrm{~m}$ de atitude) e está inserido em uma matriz suburbana, que apresenta a segunda maior densidade demográfica do município. Esse fragmento tem $39 \mathrm{~m}$ de largura.

Fragmento III (FIII): situa-se no centro (29³0'07.30'S e 5059'52.27' W, $388 \mathrm{~m}$ de altitude), em uma matriz urbana que apresenta a maior parte da população do município. $O$ fragmento apresenta $55 \mathrm{~m}$ de largura.

\section{Inventário florístico}

Para o levantamento dos dados florísticos nos FI, FII e FIII, respectivamente, foram realizadas excursões mensais, durante um ano, procurando-se registrar todas as espécies de samambaias (inclusive epifíticas) ocorrentes em uma área de $100 \times 10 \mathrm{~m}$, subdividida em 40 parcelas de $5 \times 5 \mathrm{~m}$ (Figura 2 ), distribuídas equitativamente em duas transecções, paralelas ao curso do rio Cadeia. O material coletado foi analisado seguindo a metodologia de

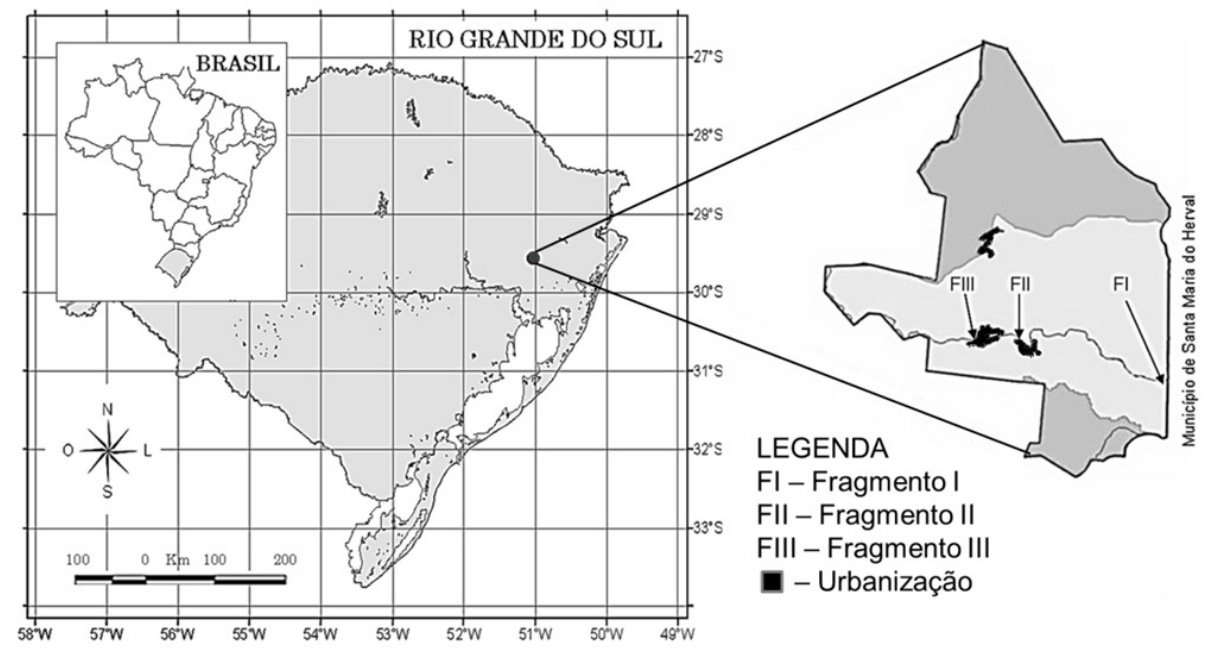

FIGURA 1: Localização dos fragmentos amostrados em Santa Maria do Herval, no estado do Rio Grande do Sul, Brasil.

FIGURE 1: Location of the sampled sites in Santa Maria do Herval, state of Rio Grande do Sul, Brazil.

\begin{tabular}{|c|c|c|c|c|c|c|c|c|c|c|c|c|c|c|c|c|c|c|c|c|}
\hline & \multicolumn{20}{|c|}{$100 \mathrm{~m}$} \\
\hline \multirow{2}{*}{$10 \mathrm{~m}$} & 1 & 2 & 3 & 4 & 5 & 6 & 7 & 8 & 9 & 10 & 11 & 12 & 13 & \begin{tabular}{l|l|}
14 &
\end{tabular} & 15 & 16 & \begin{tabular}{l|}
17 \\
\end{tabular} & 18 & 19 & 20 \\
\hline & 21 & 22 & 23 & 24 & 25 & 26 & 27 & 28 & 29 & 30 & 31 & 32 & 33 & 34 & 35 & 36 & 37 & 38 & 39 & 40 \\
\hline
\end{tabular}

FIGURA 2: Área amostrada de $100 \times 10 \mathrm{~m}$, subdividida em 40 parcelas de $5 \times 5 \mathrm{~m}$.

FIGURE 2: Sampled area of $100 \times 10 \mathrm{~m}$, subdivided into 40 plots of $5 \times 5 \mathrm{~m}$. 
campo proposta por Windisch (1992). A identificação das espécies foi realizada por meio de bibliografia especializada, comparações ao material determinado em herbário e consultas a especialistas. O sistema de classificação adotado foi o de Smith et al. (2006), com modificações apresentadas em Smith et al. (2008). Espécimes representativos, férteis, foram depositados no Herbarium Anchieta (PACA), da Universidade do Vale do rio dos Sinos, em São Leopoldo, e as duplicatas, no Laboratório de Botânica, da Universidade Feevale, Novo Hamburgo, Rio Grande do Sul. As espécies foram classificadas em formas biológicas e de crescimento, segundo sistema proposto por Raunkiaer (1934), adaptado por Mueller-Dombois e Ellenberg (1974) e Senna e Waechter (1997). Quanto ao tipo de substrato, as espécies foram classificadas em: terrícola (espécie que ocorre exclusivamente no solo); hemicorticícola (espécie que fixa raiz no solo e sobe no forófito, mantendo conexão com o mesmo durante parte do seu ciclo de vida); corticícola (espécie que cresce sobre tronco de árvore); rupícola (espécie que cresce sobre rochas); e epífito de Dicksonia sellowiana Hook. (espécie que cresce sobre capa fibrosa de raízes adventícias).

Para comparação florística da composição específica total dos fragmentos estudados, foi construída uma matriz binária de dados a partir da presença ou ausência das espécies, empregando-se o índice de Jaccard, seguido de uma análise de agrupamento pelo método de associação média (UPGMA), no programa estatístico Paleontological Statistics PAST (HAMMER et al., 2001). A distribuição espacial das parcelas amostradas nos fragmentos foi avaliada por meio da aplicação da análise de escalonamento multidimensional (MDS), com matriz de similaridade calculada também pelo índice de Jaccard. Desta forma, é possível identificar dimensões significativas subjacentes a uma distribuição de dados que permitam explicar similaridades observadas entre as mensurações do fenômeno observado, no espaço original (BORG e GROENEN, 1997).

\section{Curvas de rarefação e estimativas de riqueza}

Para verificar como o número de espécies aumentou com o número de parcelas amostradas, foram construídas curvas de rarefação, baseadas na presença ou ausência das espécies, nas parcelas (GOTELLI e COLWELL, 2001) de cada fragmento e para a amostra total (FI, FII e FIII), utilizando-se o programa estatístico EstimateS 7.5 (COLWELL,
2005), sendo que a estabilização da curva foi considerada quando a mesma assumiu uma assíntota. O mesmo programa foi utilizado para realizar as estimativas de riqueza especifica por fragmento e para a amostra total, por meio de 100 reamostragens aleatórias dos dados e com estimadores não paramétricos: Chao 2, Jackknife 1 e Bootstrap que utilizam dados de presença ou ausência de espécies. Os dados de riqueza por parcela dos fragmentos foram analisados pelo teste de Kruskal-Wallis e as médias comparadas pelo teste de Student-Newmann-Keuls (SOKAL E ROHLF, 1995), ao nível de significância de $5 \%$, por meio do programa BioEstat 4.0.

\section{RESULTADOS E DISCUSSÃO}

O levantamento florístico das samambaias, nos três fragmentos de mata ciliar amostrados ( $0,3 \mathrm{ha})$, resultou num total de 40 espécies, distribuídas em 24 gêneros e 13 famílias. Não foram encontradas espécies de licófitas nesse inventário. A riqueza de samambaias é alta quando comparada com o total de espécies do grupo registrado por Athayde Filho e Windisch (2003) em mata de galeria (18 espécies) e vereda (8); A thayde Filho e Felizardo (2007) em três segmentos florestais ao longo do rio Pindaíba (25); e por Kreutz e Athayde Filho (2009) em matas de galeria do córrego Caveira (9), no Mato Grosso. No Rio Grande do Sul, Diesel e Siqueira (1991) inventariaram 24 espécies de samambaias na mata ripária do rio dos Sinos. Essa comparação direta com os resultados de riqueza específica destes outros estudos pode levar a uma conclusão espúria (BUDDLE et al., 2005), porque não foram utilizadas curvas de rarefação de espécies que permitem avaliar quanto, aproximadamente, cada um dos inventários incluiu de toda a riqueza (GOTELLI e COLWELL, 2001), nos locais estudados.

A curva de rarefação baseada em número de unidades amostrais se aproximou da assíntota para os três fragmentos analisados, bem como para a amostra total de 120 parcelas (Figuras $3 \mathrm{e}$ 4). Considerando que o registro de todas as espécies de uma área é virtualmente impossível (SANTOS, 2006), foi estimada a riqueza total e de cada um dos fragmentos de mata ciliar de modo a torná-la comparável com inventários realizados em outras localidades, futuramente, mesmo que com maior ou menor esforço de coleta, mas que utilizarem a mesma abordagem analítica. Para a amostra total, os estimadores analíticos de riqueza apontaram que poucas espécies ainda poderão ser inventariadas, 

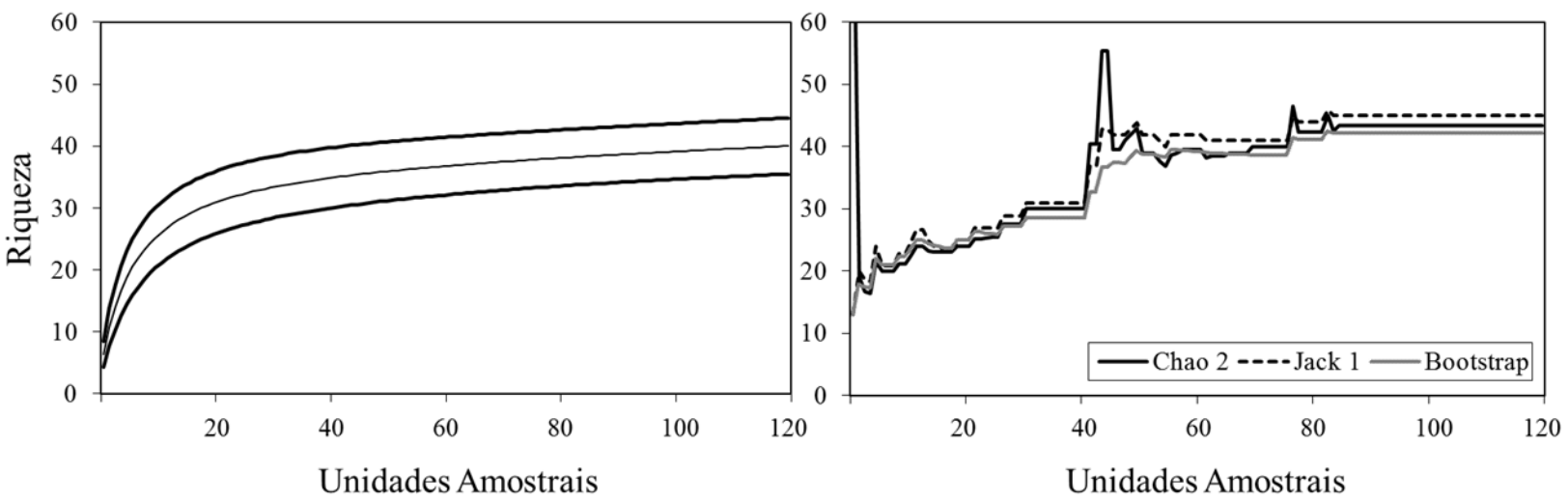

FIGURA 3: Curva de rarefação e estimativas de riqueza de espécies de samambaias para a amostra total de mata ciliar (FI, FII e FIII) do rio Cadeia, em Santa Maria do Herval, RS. Na curva de rarefação, as linhas pretas representam o intervalo de confiança.

FIGURE 3: Rarefaction curve and richness estimations of fern species for the total sample of riparian forest (FI, II and III) of river 'Cadeia', in Santa Maria do Herval, RS state. The dark lines on the rarefaction curve represent the confidence interval.
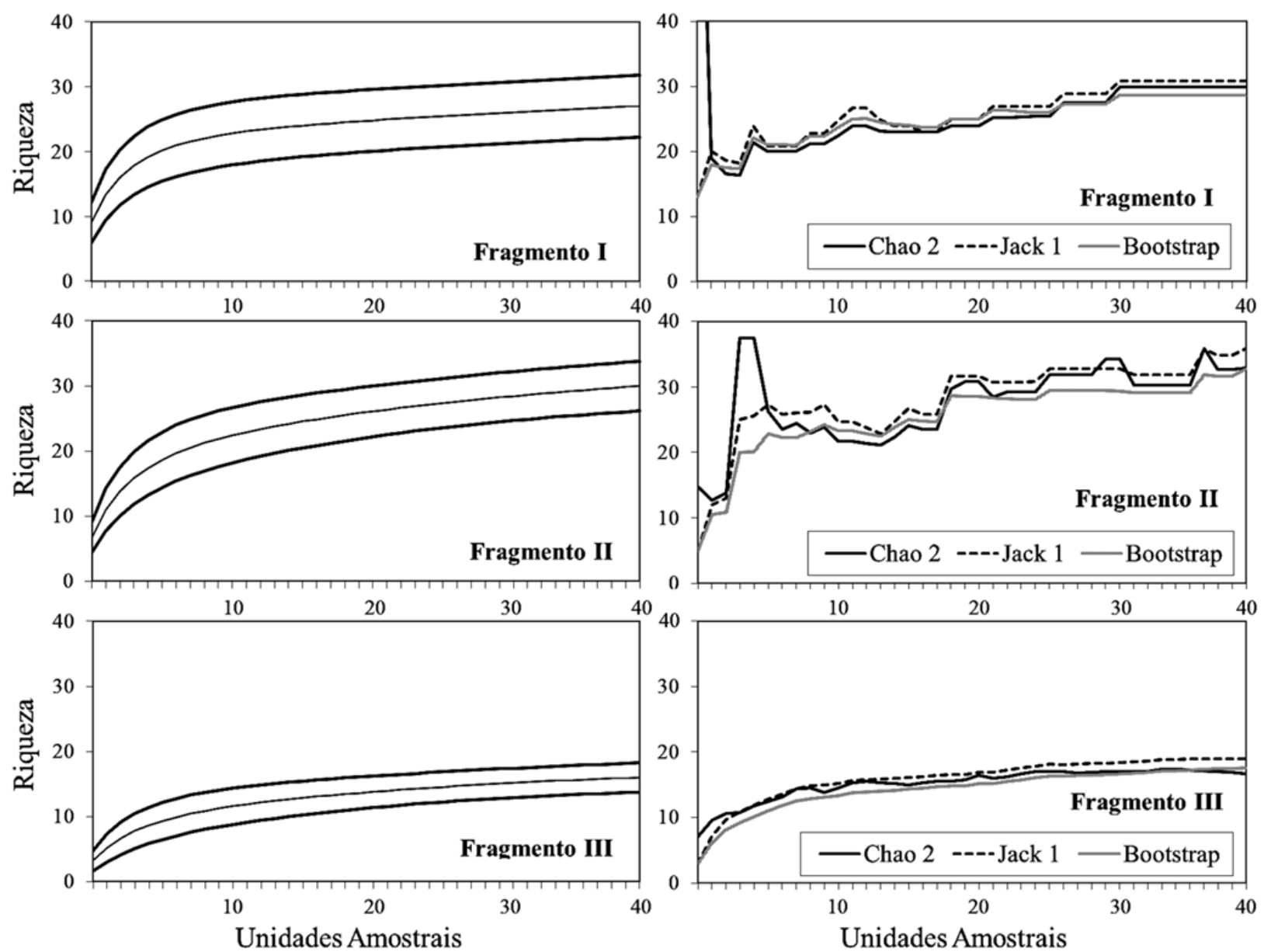

FIGURA 4: Curva de rarefação e estimativas de riqueza de espécies de samambaias dos três fragmentos de mata ciliar (FI, FII e FIII) do rio Cadeia, em Santa Maria do Herval, RS. Na curva de rarefação, as linhas pretas representam o intervalo do confiança.

FIGURE 4: Rarefaction curve and richness estimations of fern species for three fragments of riparian forest (FI, II and III) of river 'Cadeia', in Santa Maria do Herval, RS state. The dark lines on the rarefaction curve represent the confidence interval. 
sendo que foram estimadas entre 42 (Bootstrap) e 45 (Jackknife 1), indicando, respectivamente, que entre 89 e $94 \%$ da riqueza específica foi amostrada.

No FI, entre $87 \%$ e $93 \%$ das espécies de samambaias podem ter sido amostradas, sendo que foram estimadas no mínimo 29 (Bootstrap) e no máximo 31 espécies (Jackknife 1). No FII poderá ser encontrado o maior número de espécies novas, em decorrência de que os estimadores indicaram que entre $83 \%$ e $91 \%$ da riqueza específica total pode ter sido inventariada, estimando um total de 33 (Bootstrap e Chao 2) a 36 espécies (Jackknife 1). Já no FIII, os estimadores de riqueza apresentaram uma amplitude entre 17 (Chao 2 e Bootstrap) e 19 (Jackknife 1), sendo que, consequentemente, entre $84 \%$ e $94 \%$ das espécies da comunidade de samambaias devem ter sido amostrados (Figura 4). Essas estimativas de riqueza geradas não fornecem previsões precisas do número real de espécies em uma comunidade, mas apontam os valores mínimos esperados (COLWELL et al., 2004).

No estado do Mato Grosso, no município de Campinápolis, Athayde Filho e Agostinho (2005) registraram em vereda antropizada cinco espécies de samambaias, estimando a presença de mais uma espécie, baseando-se no conceito estatístico de cobertura de amostra (ICE - Incidence-based Coverage Estimator). Não foi possível realizar muitas comparações quantitativas com os resultados estimados do número de espécies de samambaias, em decorrência de que a descrição de comunidades desse grupo de plantas, em florestas ribeirinhas brasileiras, ainda não inclui, com frequência, métodos padronizados de estimativa do número de espécies. A baixa frequência de uso de estimadores de riqueza de samambaias pode estar ligada ao fato de que o seu uso em ecologia ainda é relativamente recente (SANTOS, 2006).

A média de riqueza de espécies diferiu significativamente entre os fragmentos de mata ciliar do rio Cadeia, no município de Santa Maria do Herval (Tabela 1). O FI apresentou as maiores médias de espécies herbáceas, epifíticas e totais por parcela e o FIII as menores. O FI, além de estar imerso em uma matriz rural, não sofreu ação antrópica intensa num passado recente, o que pode estar elevando a riqueza média por área florestal. Por outro lado, o FII já está imerso em uma matriz suburbana e, na área amostrada, foram encontrados 26 indivíduos adultos de árvores exóticas, incluindo espécies de uva japonesa, ligustro, pinus e eucalipto (MALLMANN, 2009). As espécies exóticas são consideradas uma das maiores ameaças à biodiversidade, que resultam da atividade humana no mundo inteiro (PRIMACK e RODRIGUES, 2001), sendo um importante agente de mudança estrutural da paisagem florestal do rio Cadeia.

Especificamente, em relação riqueza média de epífitos, o ritidoma liso de algumas árvores exóticas como o do eucalipto, retém menos água, impedindo ou dificultando a germinação de esporos e, posteriormente, o estabelecimento de esporófitos de samambaias. Kernan e Fowler (1995) observaram que a textura do substrato pode afetar a capacidade de um epífito de colonizar um forófito. A riqueza média de epífitos maior encontrada no FI sugere que o mesmo é o mais antigo das áreas amostradas, devido ao fato de que as florestas secundárias iniciais e tardias apresentam redução na riqueza de epífitos (BUDOWSKI, 1965; PINTO et al., 1995; BARTHLOT et al., 2001), sendo que o tempo de disponibilidade do substrato é fator importante para a

TABELA 1: Riqueza (S) média de espécies de samambaias herbáceas, epifíticas e totais por parcela, com seu respectivo desvio-padrão (DP), em três fragmentos de mata ciliar (FI, FII e FIII) do rio Cadeia, em Santa Maria do Herval, RS. Médias com mesma letra são iguais entre si pelo teste de Student-Newmann-Keuls ao nível de significância de 5\%.

TABLE 1: Average richness (S) of herbaceous, epiphytic and total ferns per sample unit, with the respective standard deviation (DP), in three fragments of riparian forest (FI, II and III) of river 'Cadeia', in Santa Maria do Herval, RS state. The average numbers with the same letter are equal by Student-Newmann-Keuls test, with a significance level of $5 \%$.

\begin{tabular}{cccc}
\hline Fragmentos & $\begin{array}{c}\text { S Herbáceas } \\
\text { (DP) }\end{array}$ & $\begin{array}{c}\text { S Epífitos } \\
\text { (DP) }\end{array}$ & S total (DP) \\
\hline FI & $5,9 \pm 1,7$ (a) & $3,3 \pm 1,9$ (a) & $9,1 \pm 2,5$ (a) \\
FII & $4,7 \pm 2,3$ (b) & $2,3 \pm 2,6$ (b) & $6,8 \pm 3,2$ (b) \\
FIII & $1,9 \pm 1,1$ (c) & $1,2 \pm 1,8$ (b) & $3,2 \pm 1,8$ (c) \\
\hline Kruskal- & 62,7 & 19,6 & 60,7 \\
Wallis (H) & & & \\
& $\mathrm{P}<0,001$ & $\mathrm{P}<0,001$ & $\mathrm{P}<0,001$ \\
\hline
\end{tabular}


colonização dos forófitos pelos mesmos (YEATON e GALDSTONE, 1982).

$\mathrm{O}$ fato das menores médias de riqueza serem encontradas no FIII indicou um grau de conservação ainda menor e pode ser reflexo de maior interferência antrópica, uma vez que essa área insere-se em uma matriz urbana. Somado a isso, dados históricos, da década de 40, apontam que grande parte da mata ciliar primária foi removida para a construção de uma barragem nesse ponto do rio Cadeia.

$\mathrm{Na}$ análise de agrupamento, com os dados de presença e ausência do montante total de espécies de cada fragmento, a maior similaridade florística $(47 \%)$ ocorreu entre a composição específica do FI com a do FII (Figura 5), sendo que esses dois habitat compartilharam 17 espécies. Índices de similaridade menores foram registrados por Athayde Filho e Windisch (2003) entre a composição específica da comunidade de samambaias e licófitas presente em mata de galeria e vereda $(34,5 \%)$ da Reserva Biológica Mário Viana, bem como por Athayde Filho e Agostinho (2005) entre duas veredas com diferentes estados de conservação (30,8\%), em Campinápolis, Mato Grosso.

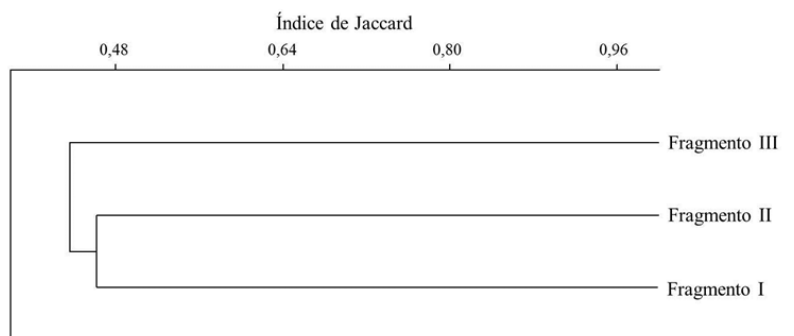

FIGURA 5: Dendograma de similaridade florística dos três fragmentos de mata ciliar (FI, FII e FIII) do rio Cadeia, em Santa Maria do Herval, RS.

FIGURE 5: Floristic similarity dendogram of three fragments of riparian forest (FI, II and III) of river 'Cadeia', in Santa Maria do Herval, RS state.

$\mathrm{Na}$ análise de escalonamento multidimensional (MDS), as parcelas do FIII ficaram ordenadas entre as do FI e FII, revelando uma falta de identidade florística própria, devido ao fato de apresentar exclusivamente uma espécie (Figura 6). As parcelas do FI formaram um agrupamento mais definido, em decorrência de que este apresentou o maior número de espécies exclusivas (10) e, consequentemente, uma composição florística mais característica, em relação aos demais fragmentos. O segundo agrupamento melhor definido foi o do FII onde foram encontradas oito espécies exclusivas. Das 40 espécies que ocorreram nos três fragmentos (Tabela 2), 12 foram comuns a todas as áreas, de modo que a ordenação produzida não distinguiu satisfatoriamente esses três grupos, que se sobrepuseram parcialmente entre si. Essas espécies generalistas, que ocuparam um maior número de ambientes, provavelmente, utilizam mais tipos de recursos e conseguem encontrá-los com maior facilidade (VENIER e FAHRIG, 1996).

A família melhor representada quanto à riqueza específica foi Polypodiaceae (11 espécies), seguida de Thelypteridaceae (seis espécies) e Dryopteridaceae (cinco espécies). Estas três famílias incluíram 55\% do total de espécies (Tabela 2). Pelo menos uma delas está entre aquelas de maior riqueza nos levantamentos realizados, no Mato Grosso, por Athayde Filho e Windisch (2003), Athayde Filho e Agostinho (2005) e Athayde Filho e Felizardo (2007); e por Rodrigues et al. (2004), no Pará. O gênero mais representativo foi Thelypteris com cinco espécies. Da mesma forma que as famílias, esse gênero está entre aqueles mais ricos registrados por Athayde Filho e Windisch (2003), Athayde Filho e Agostinho (2005) e Rodrigues et al. (2004).

A classificação das espécies quanto à sua forma de vida e de crescimento indicou a ocorrência de oito categorias, considerando todos os fragmentos. A forma biológica hemicriptófita apresentou a maior riqueza específica (19), sendo que as espécies de crescimento rosulado (14) predominaram sobre a de reptante (5). A segunda categoria mais rica foi a de epífitas (14), sendo que espécies reptantes foram as mais numerosas (13). Em seguida, foram registradas fanerófitas rosuladas (3), geófitas rizomatosas (2), caméfita rosulada e hemiepífita escandente, ambas com apenas uma espécie (Tabela 2). Com exceção de plantas terofíticas, foram constatadas todas as formas de vida conhecidas para as samambaias florestais, indicando que a área ainda apresenta condições ecológicas que favorecem o estabelecimento desse grupo de plantas. A forma de vida hemicriptófita é a mais comum em outras formações ribeirinhas, tal como apontado nos inventários realizados por Athayde Filho e Windisch (2003), Athayde Filho e Agostinho (2005), Athayde Filho e Felizardo (2007) e Kreutz e Athayde Filho (2009). As espécies hemicriptófitas apresentam gema de perenização em nível do solo, protegida 


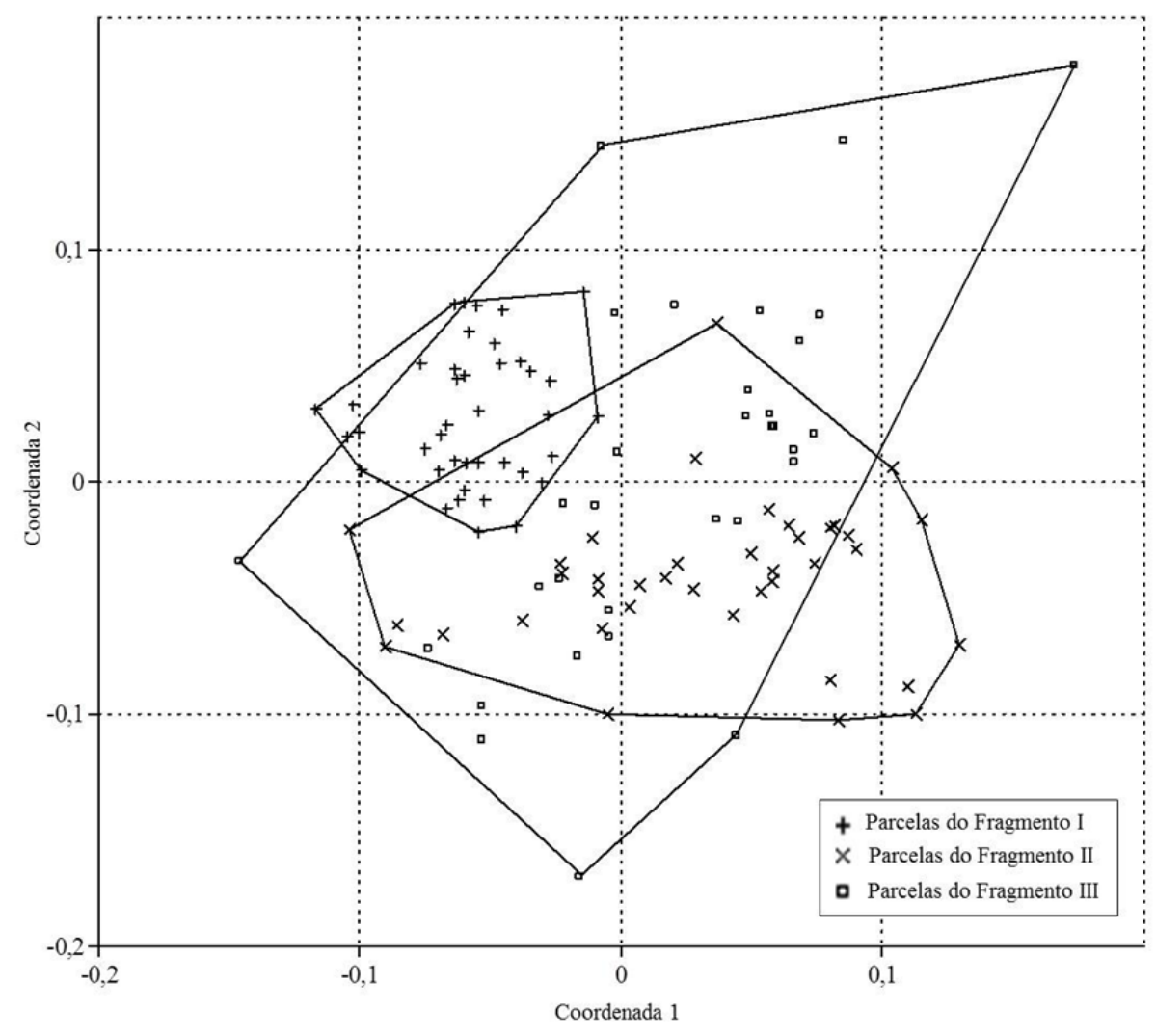

FIGURA 6: Análise de escalonamento multidimensional (MDS) gerada sobre a matriz de similaridade da comunidade de samambaias para os três fragmentos de mata ciliar (FI, FII e FIII) do rio Cadeia, em Santa Maria do Herval, RS. (Stress: 0,27).

FIGURE 6: Multidimensional scaling (MDS) analysis generated from the similarity matrix of the fern community for three fragments of riparian forest (FI, II and III) of river 'Cadeia', in Santa Maria do Herval, RS state. (Stress: 0,27).

por ele e por folhas que caem das árvores da floresta ou da própria planta, favorecendo o estabelecimento dessa forma de vida (RAUNKIAER, 1934) nos diferentes ambientes.

Polypodiaceae, que apresentou maior riqueza e exclusivamente espécies epifíticas, está entre as famílias epifíticas mais ricas da região neotropical (GENTRY e DODSON, 1987). Adaptações como poiquiloidria (BENZING, 1990) e tricomas nas folhas (MÜLLER et al., 1981) que podem completar a função de absorção realizada pelas raízes, contribuem para a ocorrência generalizada de $64 \%$ das espécies registradas para essa família, na sinúsia epifítica da mata ciliar do rio Cadeia.

Quanto ao tipo de substrato (Tabela 2), predominaram espécies exclusivamente terrícolas (19) e corticícolas (10). Da mesma forma, o predomínio de espécies terrícolas foi registrado por Athayde Filho e Agostinho (2005), Athayde Filho e Felizardo (2007) e Kreutz e Athayde Filho (2009), em diferentes formações florestais ribeirinhas. Apenas Blechnum binervatum (Poir.) C.V. Morton \& Lellinger subsp. acutum (Desv.) R.M. Tryon \& Stolze possui preferência pelo substrato hemicorticícola, sendo que a espécie germina no solo e somente depois estabelece ligação com a planta hospedeira, aparentemente, para produzir folhas férteis (DITTRICH et al., 2005).

Dentre as plantas terrícolas, foram registradas Alsophila setosa Kaulf., Cyathea atrovirens (Langsd. e Fisch.) Domin (Cyatheaceae) e Dicksonia sellowiana (Dicksoniaceae), três espécies de samambaias arborescentes, que representam alvo de extrativismo no sul do Brasil. Dicksonia sellowiana foi incluída na Lista das Espécies da Flora Brasileira Ameaçadas de Extinção (Instrução Normativa de setembro de 2008) e no apêndice II da Convenção Internacional das Espécies da Flora e da Fauna Selvagens em Perigo de Extinção (CITES), em decorrência de sua intensa exploração 
TABELA 2: Famílias e espécies de samambaias dos três fragmentos de mata ciliar (FI, FII e FIII) do rio Cadeia em Santa Maria do Herval, RS, com as respectivas formas de vida e de crescimento e tipos de substratos. Geo: Geófita; Hc: Hemicriptófita; He: Hemiepífita; Ep: Epífita; Ca: Caméfita; Fan: Fanerófita; Riz: Rizomatosa; Rep: Reptante; Esc: Escandente; Ros: Rosulada; Ter: Terrícola; Rup: Rupícola; Hco: Hemicorticícola; Cor: Corticícola; Cap: Capa fibrosa de raízes adventícias.

TABLE 2: Fern families and species from three fragments of riparian forest (FI, II and III) of river Cadeia, in Santa Maria do Herval, RS state, with the respective life and growth forms and types of substrate. Geo: geophyte; Hc: hemicryptophyte; He: hemiepiphyte; Ep: epiphyte; Cha: chamaephyte; Pha: phanerophyte; Rhi: rhizomatous; Rep: repent; Esc: Scandent; Ros: rosulate; Ter: terrestrial; Rup: rupicola; HCor: hemicorticicolous; Cor: corticicolous; Cap: fibrous adventicious root mantle.

\begin{tabular}{|c|c|c|c|c|c|}
\hline Família/espécie & $\begin{array}{l}\text { Forma Biol./ } \\
\text { Cresc. }\end{array}$ & Substrato & F I & F II & F III \\
\hline \multicolumn{6}{|l|}{ ANEMIACEAE } \\
\hline Anemia phyllitidis (L.) Sw. & Hc Ros & Ter/rup & $\mathrm{X}$ & $\mathrm{X}$ & $\mathrm{X}$ \\
\hline \multicolumn{6}{|l|}{ ASPLENIACEAE } \\
\hline Asplenium claussenii Hieron. & He Ros & Ter/Rup & $\mathrm{X}$ & & $\mathrm{X}$ \\
\hline Asplenium gastonis Fee & Ep Ros & Cor/Rup & $\mathrm{X}$ & $\mathrm{X}$ & $\mathrm{X}$ \\
\hline \multicolumn{6}{|l|}{ BLECHNACEAE } \\
\hline Blechnum binervatum (Poir.) C.V. Morton e Lellinger & He Esc & Hemcor & $\mathrm{X}$ & $\mathrm{X}$ & \\
\hline Blechnum brasiliense Desv. & Ca Ros & Ter & & $\mathrm{X}$ & \\
\hline \multicolumn{6}{|l|}{ CYATHEACEAE } \\
\hline Alsophila setosa Kaulf. & Fa Ros & Ter & & $\mathrm{X}$ & \\
\hline Cyathea atrovirens (Langsd. e Fisch.) Domin & Fa Ros & Ter & & $\mathrm{X}$ & \\
\hline \multicolumn{6}{|l|}{ DENNSTAEDTIACEAE } \\
\hline Dennstaedtia globulifera (Poir.) Hieron. & Ge Riz & Ter & $\mathrm{X}$ & $\mathrm{X}$ & $\mathrm{X}$ \\
\hline Dennstaedtia obtusifolia (Willd.) T. Moore & Ge Riz & Ter & $\mathrm{X}$ & & \\
\hline \multicolumn{6}{|l|}{ DICKSONIACEAE } \\
\hline Dicksonia sellowiana Hook. & Fa Ros & Ter & $\mathrm{X}$ & $\mathrm{X}$ & \\
\hline \multicolumn{6}{|l|}{ DRYOPTERIDACEAE } \\
\hline Ctenitis submarginalis (Langsd. e Fisch.) Ching & Hc Ros & Ter & $\mathrm{X}$ & $\mathrm{X}$ & $\mathrm{X}$ \\
\hline $\begin{array}{l}\text { Megalastrum inaequale (Kaulf. ex Link) A.R. Sm. e R.C. } \\
\text { Moran }\end{array}$ & Hc Ros & Ter & $\mathrm{X}$ & $\mathrm{X}$ & \\
\hline Polystichum longecuspis Fée & & & $\mathrm{X}$ & & \\
\hline Polystichum tijucense Fée & Hc Ros & Ter & $\mathrm{X}$ & & \\
\hline Rumohra adiantiformis (G. Forst.) Ching & Hc Rep & Ter/Cor/Cap & & $\mathrm{X}$ & \\
\hline \multicolumn{6}{|l|}{ HYMENOPHYLLACEAE } \\
\hline Trichomanes angustatum Carmich. & Ep Rep & Cap & $\mathrm{X}$ & & \\
\hline Trichomanes radicans $\mathrm{Sw}$. & Ep Rep & Cor/Rup & $\mathrm{X}$ & & \\
\hline \multicolumn{6}{|l|}{ MARATTIACEAE } \\
\hline Marattia laevis Sm. & Hc Ros & Ter & $\mathrm{X}$ & & \\
\hline \multicolumn{6}{|l|}{ POLYPODIACEAE } \\
\hline Campyloneurum austrobrasilianum (Alston) de la Sota & Ep Rep & Cor & $\mathrm{X}$ & $\mathrm{X}$ & $\mathrm{X}$ \\
\hline Campyloneurum nitidum (Kaulf.) C. Presl & Ep Rep & Cor/Rup & $\mathrm{X}$ & $\mathrm{X}$ & $\mathrm{X}$ \\
\hline Microgramma squamulosa (Kaulf.) de la Sota & Ep Rep & Cor & $\mathrm{X}$ & $\mathrm{X}$ & $\mathrm{X}$ \\
\hline
\end{tabular}


TABELA 2: Continuação ...

TABLE 2: Continued ...

\begin{tabular}{|c|c|c|c|c|c|}
\hline Família/espécie & $\begin{array}{c}\text { Forma Biol./ } \\
\text { Cresc. }\end{array}$ & Substrato & F I & F II & F III \\
\hline Microgramma vaccinifolia (Langsd. e Fisch.) Copel. & Ep Rep & Cor & & $\mathrm{X}$ & \\
\hline Niphidium rufosquamatum Lellinger & Ep Rep & Cor & $\mathrm{X}$ & $\mathrm{X}$ & $\mathrm{X}$ \\
\hline Pecluma pectinatiformis (Lindm.) M.G. Price & Ep Rep & Cor & $\mathrm{X}$ & $\mathrm{X}$ & $\mathrm{X}$ \\
\hline Pecluma truncorum (Lindm.) M.G. Price & Ep Rep & Cor & & $\mathrm{X}$ & $\mathrm{X}$ \\
\hline Pleopeltis pleopeltifolia (Raddi) Alston & Ep Rep & Cor & $\mathrm{X}$ & $\mathrm{X}$ & $\mathrm{X}$ \\
\hline Pleopeltis astrolepis (Liebm.) E. Fourn. & Ep Rep & Cor & & $\mathrm{X}$ & \\
\hline Polypodium hirsutissimum Raddi & Ep Rep & Cor & $\mathrm{X}$ & $\mathrm{X}$ & $\mathrm{X}$ \\
\hline Polypodium typicum Fée atualizar esse nome & Ep Rep & Cor & $\mathrm{X}$ & $\mathrm{X}$ & \\
\hline \multicolumn{6}{|l|}{ PTERIDACEAE } \\
\hline Adiantum raddianum C. Presl & Hc Rep & Cor/Rup & & $\mathrm{X}$ & \\
\hline Doryopteris pedata (L.) Fée & Hc Ros & Ter/cort & & $\mathrm{X}$ & \\
\hline Pteris deflexa Link & Hc Rep & TeCort & $\mathrm{X}$ & $\mathrm{X}$ & $\mathrm{X}$ \\
\hline \multicolumn{6}{|l|}{ THELYPTERIDACEAE } \\
\hline Macrothelypteris torresiana (Guadich.) Ching & Hc Ros & Ter & & $\mathrm{X}$ & \\
\hline Thelypteris dentata (Forssk.) E.P. St. John & Hc Ros & Ter & & $\mathrm{X}$ & \\
\hline Thelypteris opposita (Vahl) Ching & Hc Ros & Ter & $\mathrm{X}$ & $\mathrm{X}$ & \\
\hline Thelypteris recumbens (Rosenst.) C.F. Reed. & Hc Ros & Ter & $\mathrm{X}$ & & \\
\hline Thelypteris riograndensis (Lindm.) C.F. Reed & Hc Ros & Ter/rup & $\mathrm{X}$ & $\mathrm{X}$ & \\
\hline Thelypteris scabra (PRESL.) Lellinger & Hc Ros & Ter & & & $\mathrm{X}$ \\
\hline \multicolumn{6}{|l|}{ WOODSIACEAE } \\
\hline Diplazium petersenii (Kunze) H.Christ & Hc Rep & Ter & & $\mathrm{X}$ & $\mathrm{X}$ \\
\hline Diplazium herbaceum Fée & Hc Rep & Ter & $\mathrm{X}$ & & \\
\hline TOTAL & & & 27 & 30 & 16 \\
\hline
\end{tabular}

econômica para fins de ornamentação e paisagismo (WINDISCH, 2002).

Algumas espécies de epífitos são exclusivas ou crescem preferencialmente sobre samambaias arborescentes (FRAGA et al., 2008). Trichomanes angustatum Carmich. (Hymenophyllaceae) ocorreu exclusivamente sobre a capa fibrosa de raízes adventícias de Dicksonia sellowiana, apenas na área I. Esse epífito não foi encontrado sobre indivíduos de Dicksoniaceae e Cyatheaceae, presentes na área II. Embora, Trichomanes angustatum tenha sido registrada por Schmitt (2006) sobre Cyatheaceae, no Rio Grande do Sul, provavelmente, a espécie não ocorreu no FII, em decorrência das possíveis plantas hospedeiras medirem menos de 1,5 $\mathrm{m}$ de altura.

\section{CONCLUSÕES}

O conhecimento da riqueza da comunidade de samambaias demonstrou a importância da mata ciliar do rio Cadeia para a conservação da biodiversidade vegetal, na bacia hidrográfica do rio Caí, considerando que $12,4 \%$ do total de espécies citadas para o Rio Grande do Sul foram registradas no presente estudo. Esses resultados podem ser comparados diretamente com aqueles obtidos em outros estudos que utilizam rarefação e estimativas de riqueza, o que não é possível em muitos dos inventários realizados no Brasil.

A composição de espécies, obtida no presente inventário, é fundamental para a realização de estudos posteriores sobre as interações espaço-temporais, que ocorrem no ecossistema florestal, tornando, provavelmente, essa composição variável no tempo e no espaço.

À medida que aumenta a urbanização do habitat matriz dos fragmentos foi observado um decréscimo da riqueza média de samambaias, indicando que o aumento do grau de ação antrópica influencia negativamente a riqueza de espécies da mata ciliar. 
A conservação integral da mata ciliar do rio Cadeia conduz à manutenção de um maior número de espécies, porque os fragmentos não são homogêneos quanto à sua composição específica. Independentemente de eles apresentarem algum tipo de alteração de origem antrópica, todos devem ser considerados no estabelecimento de futuras medidas de manejo e conservação.

\section{AGRADECIMENTOS}

A realização deste estudo somente foi possível mediante o apoio e infraestrutura concedidos pela Universidade Feevale e a autorização dos proprietários das áreas para a realização dos trabalhos de campo. Acadêmicos do Curso de Ciências Biológicas da Universidade Feevale prestaram um auxílio de grande importância durante a execução das atividades de pesquisa.

\section{REFERÊNCIAS BIBLIOGRÁFICAS}

ATHAYDE FILHO, F. P.; AGOSTINHO, A. A. Pteridoflora de duas veredas no município de Campinápolis, Mato Grosso, Brasil. Pesquisas, Botânica, São Leopoldo v.56, p.145-160, 2005.

ATHAYDE FILHO, F. P.; WINDISCH, P. G. Análise da pteridoflora da Reserva Biológica Mário Viana, Município de Nova Xavantina, Estado de Mato Grosso (Brasil). Bradea, Rio de Janeiro, v. 9, n. 13, p. 67-76, 2003.

ATHAYDE FILHO, F. P.; FELIZARDO, M. P. P. Florística e aspectos ecológicos da pteridoflora em três segmentos florestais ao longo do rio Pindaíba, Mato Grosso. Pesquisas, Botânica, São Leopoldo, v. 58 , p. $227-244,2007$.

BARRELA, W. et al. As relações entre as matas ciliares, os rios e os peixes. In: RODRIGUES, R. R.; LEITÃO FILHO, H. F. (Eds.). Matas Ciliares: conservação e recuperação. São Paulo: EDUSP, 2000. p. 187-207.

BARTHLOT, W. et al. Diversity and abundance of vascular epiphytes: a composition of secondary vegetation and primary montane rain forest in the Venezuelan Andes. Plant Ecology, Holanda, v. 152, p. 145-156, 2001.

BENZING, D. H. Vascular epiphytes. Cambridge: Cambridge University Press, 1990. 354 p.

BORG, I.; GROENEN, P. Modern Multidimensional Scaling: Theory and Applications. Nova York: Springer, 1997. 614 p.

BUDDLE, C. M. et al. The importance and use of taxon sampling curves for comparative biodiversity research with forest arthropod assemblages. Canadian Entomologist, Canadá, v. 137, p. 120127, 2005.

BUDOWSKI, G. Distribuition of tropical american rain forest species in the light of sucessional processes. Turrialba, San José, v. 15, n. 1, p. 40-42, Jan./Mar. 1965.

CAMPOS, J. C. de; LANDGRAF, P. R. C. Análise da regeneração natural de espécies florestais em matas ciliares de acordo com a distância da margem do lago. Ciência Florestal, Santa Maria, v. 11, n. 2, p. 143-151, 2001.

COLWELL, R. K. Estimates: Statistical estimation of species richness and shared species from samples. Versão 7.5. Guía de usuário e aplicativo, 2005. Disponível em: http://viceroy.eeb.uconn.edu/ EstimateS

COLWELL, R. K. et al. Interpolatin, extrapolatin, and comparing incidence-based species accumulation curves. Ecology, Londres, v. 85, p. 2717-2727, 2004.

DIESEL, S.; SIQUEIRA, J. C. Estudo fitossociológico herbáceo/arbustivo da mata ripária da bacia hidrográfica do rio dos Sinos, Rio Grande do Sul. Pesquisas, Botânica, São Leopoldo, v. 42, n. 2, p. 205-257, 1991.

DITTRICH, V. A. O. et al. Species richness of pteridophytes in a montane Atlantic rain forest plot of Southern Brazil. Acta Botanica Brasílica, São Paulo, v. 19, n. 3, p. 519-525, 2005.

FALAVIGNA, T. J. Diversidade, formas de vida e distribuição altitudinal das pteridófitas do Parque da Ferradura, Canela (RS), Brasil. 2002. 90 f. Dissertação (Mestrado em Biologia) - Universidade do Vale do Rio dos Sinos, São Leopoldo, 2002.

FRAGA, L. L. et al. Composição e distribuição vertical de pteridófitas epifíticas sobre Dicksonia sellowiana Hook. (Dicksoniaceae), em floresta ombrófila mista no sul do Brasil. Biota Neotropica, v. 8, n. 4, p. 123-129, out./dez. 2008.

GENTRY, A. H.; DODSON, C. Contribuition of nontrees to species richness of a tropical rain forest. Biotropica, Washington, v. 19, n. 2, p. 149-156, 1987.

GOTELLI, N. J.; COLWELL, R. K. Quantifying biodiversity: procedures and pitfalls in the measurement and comparison of species richness. Ecology Letters, Boston, v. 4, p. 379-391, 2001. GREGORY, S. V. et al. An ecosystem perspective of riparian zones. BioScience, Minas Gerais, v. 41, n. 
8, p. $540-551,1992$.

HAMMER, Ø. et al. PAST: Paleontological Statistics Software Package for Education and Data Analysis. Palaeontologia Electronica, v. 4, n. 1, p. 1-9, 2001.

KERNAN, C.; FOWLER, N. Differential substrate use by epiphytes in Corcovado National Park, Costa Rica: a source of guild structure. Journal of Ecology, Oxford, v. 83, n. 1, p. 65-73, 1995.

KREUTZ, C.; ATHAYDE FILHO, F. P. Aspectos Ecológicos da Flora Pteridofítica do Córrego Caveira, Barra do Garças - MT. In: CONGRESSO DE ECOLOGIA DO BRASIL, 9., 2009, São Lourenço. Anais ..., São Lourenço, 2009, p. 1-4. LORSCHEITTER, M. L. et al. Pteridophyte spores of Rio Grande do Sul flora, Brazil. Part I. Palaeontographica Abt. B, Stuttgart, v. 246, p. 1-113, 1998.

LORSCHEITTER, M. L. et al. Pteridophyte spores of Rio Grande do Sul flora, Brazil. Part VI. Palaeontographica Abt. B, Stuttgart, v. 281, p. 1-96, 2009.

MALLMANN, I. T. Comparação da composição florística, estrutura comunitária e distribuição espacial de monilófitas entre três fragmentos de mata ciliar do rio Cadeia, com diferentes graus de perturbação antrópica, no sul do Brasil. 2009. 76 f. Dissertação (Mestrado em Qualidade Ambiental) - Universidade Feevale, Novo Hamburgo, 2009.

MOREnO, J. A. Clima do Rio Grande do Sul. Porto Alegre: Governo do Estado do Rio Grande do Sul - Secretaria da Agricultura, 1961. 41 p.

MUELLER, C. C. Gestão de matas ciliares. In: LOPES, I. V. (org.). Gestão Ambiental no Brasil: experiência e sucesso. Rio de Janeiro: Fundação Getúlio Vargas, 1996. p. 185-214.

MUELLER-DOMBOIS, D.; ELLENBERG, G. H. Aims and methods of vegetation ecology. New York: Wylley e Sons, 1974. 547 p.

MÜLLER, L. et al. Zur Ökologie epiphytisher Farne in Südbrasilien. I. Saugschuppen. Flora, Jena, v. 171, p. 55-63, 1981.

OLIVEIRA-FILHO, A. T.; RATTER, J. A. A study of the origin of Central Brazilian forests by the analysis of plant species distribution patterns. Edinburgh Journal of Botany, Edinburgh, v. 52, n. 2, p. 141-194, 1995.

PINTO, A. C. R. et al. Composição florística de epífitas (Magnoliophyta) em fragmento de floresta no município de Jaboticabal, SP, Brasil. Científica, v. 23, n. 2, p. 283-289, 1995.
PRADO, J.; SYLVESTRE, L. Pteridófitas. In: FORZZA, R. C. et al. (orgs). Lista de Espécies da Flora do Brasil. Rio de Janeiro: Jardim Botânico do Rio de Janeiro. 2010.

PRIMACK, R. B.; RODRIGUES E. Biologia da Conservação. Londrina: E. Rodrigues, 2001.328 p. RAUNKIAER, C. The life forms of plants and statistical geography. Claredon, Oxford, 1934. $632 \mathrm{p}$.

RIBEIRO-FILHO, A. A. Composição florística da floresta ciliar do rio Mandassaia, Parque Nacional da Chapada Diamantina, Bahia, Brasil. Rodriguésia, Rio de Janeiro, v. 60, n. 2, p. 265-276, 2009.

RODRIGUES, R. R.; NAVE, A. Heterogeneidade florística das matas ciliares. In: RODRIGUES, R. R.; LEITÃO FILHO, H. de F. (Org.). Matas ciliares: conservação e recuperação. São Paulo, Edusp: FAPESP, 2001. p. 45-71.

RODRIGUES, R. R.; GANDOLFI, S. Conceitos, tendências e ações para a recuperação de florestas ciliares. In: RODRIGUES, R. R.; LEITÃO FILHO, H. de F. (Org.). Matas Ciliares: conservação e recuperação. São Paulo, Edusp: FAPESP, 2000. p.233-247.

RODRIGUES, S. T. et al. Composição florística e abundância de pteridófitas em três ambientes da bacia do rio Guamá, Belém, Pará, Brasil. Acta Amazonica, Manaus, v. 34, n. 1, p. 35-42, 2004.

SANTOS, J. Estimativas de riqueza em espécies. In: CULLEN Jr. L. et al., (eds.). Métodos de Estudos em Biologia da Conservação e Manejo da Vida Silvestre. Curitiba: Ed. da UFPR, 2006. p.19 - 41. SARMENTO, E. C. et al. Avaliação da cobertura vegetal na microbacia Feitoria/Cadeia utilizando técnicas de geoprocessamento. 2001. Disponível em: http://www.ecologia.ufrgs.br/labgeo/artigos/ cadeia.pdf (acessado em 06/06/2007).

SCHMITT, J. L. Composição Florística e Ecologia de Pteridófitas Epifíticas em Cyatheaceae no estado do Rio Grande do Sul, Brasil. In: MARIATH, J. E. de A.; SANTOS, R. P. dos (Orgs.). Os avanços da Botânica no início do século XXI: morfologia, fisiologia, taxonomia, ecologia e genética: Conferências Plenárias e Simpósios do $57^{\circ}$ Congresso Nacional de Botânica. Porto Alegre: Sociedade Botânica do Brasil, 2006. p. 336-339.

SEHNEM, A. Semelhanças e diferenças nas formações florestais do Sul do Brasil. Acta Biológica Leopoldensia, São Leopoldo, v. 1, p. 111-135, 1979.

SENNA, R. M.; WAECHTER, J. L. Pteridófitas de uma Floresta de Araucária. 1. Formas Biológicas 
e padrões de distribuição geográfica. Iheringia Serie Botânica, Porto Alegre, v. 48, p. 41-58, 1997. SMITH A. R. et al. A classification for extant ferns. Taxon, Vienna, v. 55, n. 3, p. 705-731, Aug. 2006. SMITH, A. R. et al. Fern classification. In: Ranker, T. A.; Haufler, C. H. (eds) Biology and evolution of fern and Lycophytes. Cambridge: Cambridge University Press, p. 417-467. 2008.

SOKAL, R. R; ROHLF, F. J. 1995. Biometry: the principles and practice of statistics in biological research. 3rd ed. New York: W.H. Freeman and Co. $887 \mathrm{p}$.

SONEGO, R. C. et al. Descrição da estrutura de uma Floresta Ombrófila Mista, RS, Brasil, utilizando estimadores não-paramétricos de riqueza e rarefação de amostras. Acta Botanica Brasilica, São Paulo, v. 21, n. 4, p. 943-955, 2007.

TEIXEIRA, M. B.; MOURA NETO, A. B. C. As regiões fitoecológicas, sua natureza e seus recursos econômicos - Estudo Fitogeográfico. Rio de Janeiro: Projeto RADAMBRASIL, 1986. p.
791. V. 33

VENIER L. A.; FARIG L. Habitat availability causes the species abundance distribution relationship. Oikos, Lund, v. 76, n. 3, p. 564-570, 1996.

WINDISCH, P. G. Pteridófitas da Região Norte-Ocidental do Estado de São Paulo Guia para excursões. 2. ed. São José do Rio Preto: Universidade Estadual Paulista, 1992. 110 p.

WINDISCH, P. G. Towards assaying biodiversity in Brazilian pteridophytes. In: BICUDO, C. E. M.; MENEZES, N. A. Biodiversity in Brazil: a first approach. São Paulo: CNPq. 1996. p. 109-117. WINDISCH, P. G. Fern conservation in Brazil. Fern Gazette, Australia, v. 16, p. 295-300, 2002. YEATON, R. I.; GLADSTONE, D. E. The pattern of colonization of epiphytes on Calabash Trees (Crescentia alata HBK.) in Guanacaste Province, Costa Rica. Biotropica, v. 14, p. 137-140, 1982.

ZAR, J. H. Biostatistical Analysis. 4th ed. Upper Saddle River: Prentice-Hall, 1999. 929 p. 